\title{
On Timoshenko's Beams Coefficient of Sensibility to Shear Effect
}

F. PIETROBON COSTA ${ }^{1}$, Computational and Applied Mathematics Group, Exact
Sciences and Technology Department, DCET, UESC, 45662-000 Ilheus, BA, Brasil.

\begin{abstract}
Classical beams theory usually neglect shear contribution to deformation. Timoshenko's Beam Theory (TBT) corrects this negligence. This work is a step in the research to check accuracy of TBT in piezoactuators and in nano beams, particularly in Carbon nanotubes (CNT). Before immerse into the nanoscale problem, the superior limit of applicability of TBT must be investigated. This work introduces a proposition to the range of validation of TBT. A sensibility coefficient related to free vibratory beams is proposed, in terms of a ratio of deflected geometry to aspect ratio of the beams. Finite difference and Galerkin finite element were used to formulate the computational model. This approach was checked with experimental results to propose a top validation of TBT. Convergence in solution process was verified with precedent works. Results are related to symmetric dynamic bending of beams considering shear deformability and rotatory inertia, at small change of configuration. Timoshenko's beam models show a displacement field greater than those obtained with Euler-Bernoulli Theory (EBT). The magnitude of the difference is of 6 to $34 \%$ greater in TBT in relation to EBT, for tree kinds of boundary conditions, for the same beam geometry and load pattern.
\end{abstract}

\section{Introduction}

The precision in the determination of the limit of validity between Timoshenko (TBT) and Euler-Bernoulli Theories (EBT) is associated to the amplitude of applications and dynamic responses in vibratory bars. Both TBT and EBT scout solutions to problems related with shear effect.

\subsection{State of the art}

Since Timoshenko's [23] work on bending of beams, and Reissner [19] and Mindlin [15] works in shear deformation of plates, shear deformability has been a well succeed research line. Timoshenko proposed a shear correction factor, k. This factor is a compatibility criterion between real shear stress and distortion of beams. Timoshenko introduces this factor, in beam theory, in order to account with warping and distortion of transversal (or as referred in classical literature: cross) section. Cowper [4] studied the influence of shear coefficient, obtained by means

\footnotetext{
${ }^{1}$ pietrobon_costa@yahoo.com.br
} 
of three dimensional elasticity equations. Levinson and Cook, [13], [14], proposed new formulations to shear coefficient, considering that plane sections, normal to beam longitudinal axis, in the non deformed configuration, becomes curve and deformed, after bending. The importance of TBT is clarified when the diversity of works using it are considered, as the work of Beran [3] who discussed the question of micro-beams composed of polycrystals, which dimensions are of the order of transversal beams dimensions, and as Katori [11], considering the use of EBT formulation in shear and torsion analysis. More recently, Wang [24] considered the use of the Timoshenko beam model for free vibration analysis of multi-walled carbon nanotubes (CNTs). Those two last works introduces two questions: (1st) about how and when Timoshenko's (TBT) and classical Euler beam (EBT) theories have limits of validity between them, and (2nd) if there is a range of application of TBT in the nanoscale problem. Those questions are present in the studies of smart and composite structures as done by Krommer and Irschik [12] in the study of coupled electrical-mechanical fields to piezoelectric sensors and actuators, and in the work of Sun, Tong and Atturi [22] that investigated the efficiency of vibration control in smart structures. The greater accuracy of TBT, especially for higher frequencies, when compared with the solutions developed with the employment of EBT, is a goal for systems of beams, like frames, as Antes et al. [1] detected. The work of Formaggia et al. [6] increased the amplitude of applications of TBT, beeing an application of the shear correction factor to compute radial deformation of a artery compliant tube. Arnold, Madureira and Zhang [2] studied the range of applicability of Reissner-Mindlin, the TBT version in 2D and 3D, in 3D thin plate elastic models. This is one of the few works that try to determine a range of validity to the theory. In an analogous way, Harik [9] proposed ranges of validity of the continuum beam model for carbon nanotubes (CNT).

\subsection{TBT application in CNT}

For micro and nano beams a small configuration change assumption, coupled with linear elasticity, results in the validation of Hooke's Law for the formulation of the problem. Harik, Wang, and Pietrobon ([16] and [17]) had already validate this assumption; the two formers for CNT and smart beams. Carbon nanotubes (CNT) are a way to realize extreme properties and benefits in stiffness and strength-to-weight high ratios. CNT can generate rods or beams for applications in nanomachines and nanostructures [7]. CNT generated interesting material investigations because of high strength and stiffness in comparison to others materials. Some structural aspects and behavior of CNT remains not well known. One of them is which theory is the better one. EBT has been used to simulated CNT beams, but this theory do not describes well the shear deformability. In TBT a growth in the deformability values, relatively to longitudinal axis, is the result of shear effect in beams. This behavior is more precise when compared with the classical one, obtained with EBT. According to Harik [9] the Hypothesis of Continuum has validity for CNT beams if the ratios $\left(1^{s t}\right)$ characteristic dimension $\left(d_{N T}\right.$ or just $\left.\mathrm{h}\right)$ of the transversal section to wall width $\left(a_{w}\right)$ and $\left(2^{n d}\right)$ aspect ratio, the ratio of characteristic dimension, $\mathrm{h}$, 
to length, L, satisfied:

$$
\begin{aligned}
& \frac{h}{a_{w}} \approx 1, \\
& \frac{h}{L}<<1 .
\end{aligned}
$$

For the objectives of this work that hypothesis was adopted and the analysis was restricted for an aspect ratio of 0.250 or lesser. This work postulate that TBT is a better beam theory for shear effect consideration in micro-beams and in CNT nano-beams. This growth in deformation and distortion of the transversal section, or shear effect, is usually neglected when EBT is used, but the frontier for nonconsideration of shear effect contributions is yet not well posed. The analysis of this work is focused on an effort to determine a superior limit of validity to TBT. This is a first step in TBT application to nano beams simulations.

\section{Conceptual Model}

I take into account symmetrical bending of beams with little change of configurations. I consider also a corrected rotation of transversal section in coupled bending and shear effect analysis. Numerical solution was obtained in an energetic approach, applied with the minimizations of the functional of minimal potential energy, the Hamilton's Principle ([20], [8], and [5]). That approach requests only prescription of Dirichlet conditions, resulting for solid mechanics, in symmetry for bandwidth matrix of coefficients. A semi-implicit finite differences (FDM) formulation was employed to deal with the time derivatives associated with the acceleration, as used before in early works [16] with success. A formulation in finite elements was also developed to deal with spatial terms, in an classical Galerkin approach. To solve numeric models of Timoshenko beams I adopt models with formal boundary conditions and geometric patterns, to determine a coefficient sensibility to shear deformability effect. At this base a computational model was implemented and solved numerically. The results obtained allow the proposition of a beam sensibility coefficient to shear deformability, as a function of geometric and deformed pattern of the beam. Results were obtained to three models, in relation to the boundary conditions: (ss) simple supported in both ends (hinged), (sc) hinged in one end and fixed (or clamped) in the other one, and (cc) fixed in both ends. One dimensional beam models were used. Responses were plotted in transversal displacement ratio, $\mathrm{w}(\mathrm{G} 0) / \mathrm{w}(\mathrm{G} 4)$, versus aspect ratio, $\mathrm{h} / \mathrm{L}$. Transversal displacement ratio relates transversal displacement, with, w(G0), and without, w(G4), consideration of shear effect. That formulation allow a definition of a sensibility coefficient to shear effect in Timoshenko beams - $\eta$ - as a ratio between differentials of non dimensional displacement and aspect ratio,

$$
\eta=\frac{\delta \frac{w(G 0)}{w(G 4)}}{\delta \frac{h}{L}} .
$$

In this way it's possible also to determine the influences of boundary conditions. Results were obtained taking in account a dynamic equilibrium condition for the 
structural system. Small configuration changes induce linear geometric behavior. This work do not consider the effect of damping in the dynamic answer. In this work the following assumptions were posed to solve the problem: (1) loads acts transversally, time independent, in the plan of symmetry xz; (2) the material is homogeneous, isotropic and linearly elastic; (3) normal stresses in y and z directions are small compared to the normal stress $\mathrm{x}$; (4) the transversal displacement, w, act transversally to longitudinal axis of the beam; (5) sections that are plane and normal to the beam longitudinal axis, in the non-deformed configuration, come plane and non-deformed, but not normal to the axis, after the bending.

\section{Variational Formulation}

A displacement field, $\mathbf{u}$ is considered. Assumption 5 allows the considerations of a strain-displacement relation. Transversal sections suffer only an angle rotation, $\psi$, the beam longitudinal axis rotation angle, not equal to the declivity $\partial \mathrm{w} / \partial \mathrm{x}$ of longitudinal axis. In the scope of the Linear Theory of the Elasticity that relation between differential operator $\mathbf{B}$ and displacement field becomes

$$
\epsilon=\mathbf{B u} \text {. }
$$

Hooke's Law is derived from this last relation as a stress-strain relation, or a stressdisplacement one, where $\mathbf{D}$ is the elasticity matrix, in the form:

$$
\sigma=\mathbf{D} \epsilon
$$

or

$$
\sigma=\mathbf{D B u} .
$$

Displacement field, differential operator, and elasticity matrix are given respectively as in Pietrobon [16].

An action functional, $\phi(\mathbf{u}, \mathrm{t})$, is developed from Hamilton's Principle, considering kinetic, deformation and potential (from external loads) energies, respectively $\mathrm{T}, \mathrm{U}$ and $\mathrm{P}$, between $\mathrm{t} 1$ and $\mathrm{t} 2$ time steps:

$$
\phi(\mathbf{u}, t)=\int_{t 1}^{t 2}(T-U-P) d t
$$

the minimimal form of the action functional, gives the condition of dynamic stability of the structural system, so,

$$
\delta(\phi\{\mathbf{u}, t))=0 .
$$

With that consideration equation (3.4) becomes

$$
\int_{t 1}^{t 2}\left(\int_{\Omega} \rho \dot{\mathbf{u}} \delta \dot{\mathbf{u}} d A d x+\int_{\Omega} \mathbf{u}^{T} \mathbf{B}^{T} \mathbf{D B} \delta \mathbf{u} d A d x-\int_{\Omega} \mathbf{p}^{T} \delta \mathbf{u} d A d x\right) d t=0
$$

for $\mathrm{t}=[\mathrm{t} 1, \mathrm{t} 2], \Omega$ the geometric domain that reduces to transversal section area, given by $\mathrm{A}$, plus longitudinal dimension $\mathrm{x}=[0, \mathrm{~L}]$ where $\mathrm{L}$ is the beam length, $\rho$ 
the specific density, and $\mathbf{p}$ the vector of applied external loads. After integrating by parts the terms of velocity, for arbitrary 1 st variation of $\dot{\mathbf{u}}$, in $\mathrm{t} 1$ and $\mathrm{t} 2$, and integrating by parts again the terms of first space derivatives, for I being the moment of inertia. The strong form, or differential, of the system of equations becomes

$$
\begin{gathered}
\rho A \ddot{w}-k G A\left(\frac{\partial^{2} w}{\partial x^{2}}-\frac{\partial \psi}{\partial x}\right)-p=0, \\
\rho I \ddot{\psi}-k G A\left(\frac{\partial w}{\partial x}-\psi\right)=0,
\end{gathered}
$$

considering first variations of the unknowns as arbitrary at first and final time steps. That result is exactly the same as obtained by Silva [21].

\section{Finite Differences, Numeric Scheme}

A semi-implicit finite difference scheme is adopted for odd time derivatives. This scheme is relative to the evolution of the problem in time. Consider $f$ as a general designation for the unknowns, $n$ refered to time step, and subindex related to generalized i-th discrete node segment. In this way the scheme becomes

$$
\begin{gathered}
\ddot{f}_{i}^{n}=\frac{4 f_{i}^{n}}{\Delta t^{2}}-4 \frac{\left(f_{i}^{n-1}+\dot{f}_{i}^{n-1} \Delta t\right)}{\Delta t^{2}}-\ddot{f}_{i}^{n-1}, \\
\dot{f}_{i}^{n}=\dot{f}_{i}^{n-1}+\frac{\Delta t}{2}\left(\ddot{f}_{i}^{n-1}+\ddot{f}_{i}^{n}\right) .
\end{gathered}
$$

The use of this time reduced integration model in the space of finite difference form, as employed, allows successfully convergence to the problem solution, with an error of $2^{\text {nd }}$ order in the time approximation. This approach needs an evaluation of a initial value to the second time derivative of $f$ function, a imposed initial condition. That evaluation were done with employment of a consistent approach related to Newton's Law, a mass conservative approach, where

$$
\begin{gathered}
\ddot{w}\left(x_{i}, 0\right)=\frac{p\left(x_{i}\right)}{\mu A}, \\
\ddot{\psi}\left(x_{i}, 0\right)=0, \\
\dot{w}\left(x_{i}, 0\right)=0, \\
\dot{\psi}\left(x_{i}, 0\right)=0 .
\end{gathered}
$$

\section{Finite Element, Galerkin Formulation}

A finite element formulation was developed, based in the Galerkin approach, semidiscrete in time, defining spaces for trial, $\mathbf{u}$, and weighting, $\mathbf{w}$, functions

$$
U=\left\{\mathbf{u} \in\left[H^{1}\right]^{3} \mid \mathbf{u}=\overline{\mathbf{u}} \in \partial \Omega\right\},
$$




$$
\begin{gathered}
V=\left\{\mathbf{v} \in\left[H_{0}^{1}\right]^{3} \mid \mathbf{v}=0 \in \partial \Omega\right\}, \\
\mathbf{u}=\mathbf{v}+\overline{\mathbf{u}} \mid \mathbf{v} \in V,
\end{gathered}
$$

this FEM formulation gives for finite dimensional functions

$$
\begin{aligned}
\mathbf{u}^{h} & =\sum N_{i} u_{i j}, \\
\mathbf{v}^{h} & =\sum N_{i} v_{i j},
\end{aligned}
$$

for $i=1 . . n n o, j=1 . . n g l$, where $n n o$ : number of nodes $(=\mathrm{a})$ and $n g l$ : number degrees of freedom $(=\mathrm{b})$. This formulation, applied to variational formulation, holds at each $n$ time step:

$$
\begin{gathered}
\sum_{i=1}^{a} \mathbf{v}_{i} \sum_{j=1}^{b} \int_{x_{1}}^{x_{2}}\left(\alpha \mathbf{C} N_{i} N_{j} \mathbf{u}_{i j}^{n}-\mathbf{B}^{T} \mathbf{D B} A N_{i} N_{j} \mathbf{u}_{i j}^{n-1}\right) d x^{e}= \\
\sum_{i=1}^{a} \mathbf{v}_{i} \sum_{j=1}^{b} \int_{x_{1}}^{x_{2}} \mathbf{p}^{T} N_{i} d x^{e}+\sum_{i=1}^{a} \mathbf{v}_{i} \sum_{j=1}^{b} \int_{x_{1}}^{x_{2}}\left(\alpha \mathbf{C} N_{i} N_{j} \mathbf{u}_{i j}^{n-1}+\right. \\
\left.+\alpha \mathbf{C} N_{i} N_{j} \dot{\mathbf{u}}_{i j}^{n-1}\right) d x^{e}+\sum_{i=1}^{a} \mathbf{v}_{i} \sum_{j=1}^{b} \int_{x_{1}}^{x_{2}} N_{i} N_{j} \ddot{\mathbf{u}}_{i j}^{n-1} d x^{e}
\end{gathered}
$$

for each element in the discrete mesh, as an approximation of the continuum mathematical model. In equation (5.1) $\alpha=4 / \Delta t, \mathbf{C}$ is the matrix of mass coefficients related to transversal displacement, $w$, and transversal section angle of rotation, $\psi$. As posed by Hughes [10], the assembling of nodal contributions, related to finite elements degrees of freedom, results in obtaining the equivalent global system of equations, in matrix form, where $\mathbf{R}$ is the matrix of constrains conditions

$$
\mathbf{M} \mathbf{u}^{n}-\mathbf{K u}^{n-1}=\mathbf{F}+\mathbf{R} .
$$

The global matrixes for that FEM formulation, the mass matrix, $\mathbf{M}$, the coefficients matrix, $\mathbf{K}$, and the force matrix, $\mathbf{F}$, are respectively:

$$
\begin{gathered}
\mathbf{M}=\sum_{i=2}^{a-1} \mathbf{v}_{i} \sum_{j=1}^{b} \int_{x_{1}}^{x_{2}} \alpha \mathbf{C} N_{i} N_{j} \mathbf{u}_{i j}^{n} d x^{e}, \\
\mathbf{K}=\sum_{i=2}^{a-1} \mathbf{v}_{i} \sum_{j=1}^{b} \int_{x_{1}}^{x_{2}}\left(\mathbf{B}^{T} \mathbf{D B} A N_{i} N_{j} \mathbf{u}_{i j}^{n-1}\right) d x^{e}, \\
\mathbf{F}=\sum_{i=2}^{a-1} \mathbf{v}_{i} \sum_{j=1}^{b} \int_{x_{1}}^{x_{2}} \mathbf{p}^{T} N_{i} d x^{e}+\sum_{i=2}^{a-1} \mathbf{v}_{i} \sum_{j=1}^{b} \int_{x_{1}}^{x_{2}}\left(\alpha \mathbf{C} N_{i} N_{j} \mathbf{u}_{i j}^{n-1}+\right. \\
\left.\alpha \mathbf{C} N_{i} N_{j} \dot{\mathbf{u}}_{i j}^{n-1}\right) d x^{e}+\sum_{i=2}^{a-1} \mathbf{v}_{i} \sum_{j=1}^{b} \int_{x_{1}}^{x_{2}} N_{i} N_{j} \ddot{\mathbf{u}}_{i j}^{n-1} d x^{e} .
\end{gathered}
$$




\section{Results}

The solution of the problem was done with that computational approach, for the dynamic analysis of free vibrating Timoshenko beams models. That was obtained in two stages, first by convergence study employing simple supported and clampedsupported models of beams, determining a near optimal discretization for the beam computational model, and second by analyzing sensibility to shear effect on simple supported (ss), clamped-supported (cs) and bi-clamped (cc) models. The dynamic analysis verifies the predominance of the first natural mode of vibration, allowing the consideration of time step interval, $\Delta \mathrm{t}$, as one percent of first, $T_{1}$, mode of vibration, where $a_{1}$ natural mode period parameter, obtained by Rao [18] is relative to that mode:

$$
\begin{gathered}
T_{1}=\frac{2 \pi}{\omega_{1}}, \\
\omega_{1}=a_{1} \sqrt{\frac{E I}{\mu A L}}, \\
\Delta t=\frac{T_{1}}{100} .
\end{gathered}
$$

All of those beams models considered $20 \mathrm{~nm}$ in width versus $50 \mathrm{~nm}$ in height of beam dimensions transversal section in relation to longitudinal axis, with a elastic modulus of $200 \mathrm{GPa}$. This value is relative to essays relating Young's modulus to beam diameter for CNT [7]. The first stage was approached by refining a discrete model of the beam in segments between 8 and 140 elements, for a beam length of $300 \mathrm{~nm}$. The deflection of the beams models were computed with relation to the longitudinal beam not deformed pattern, before loading, in the node of maximum displacement: midspan position, for simple supported and bi-fixed beams, and in 38 node position (model of 98 nodes discretization) for hinged-fixed beam model. The convergence rate (table 1) is presented for (ss) discretization model, in a $\Delta=\left|w^{i}-w^{i-1}\right| /\left|w^{i-1}\right|$ norm criteria.

Table 1 - Convergence discretization procedure

\begin{tabular}{|c|c|c|}
\hline $\begin{array}{c}\text { Model : no.of } \\
\text { segments }\end{array}$ & $\begin{array}{c}\text { Transversal } \\
\text { displacement, } w\end{array}$ & $\begin{array}{c}\text { Convergence } \\
\Delta(\%)\end{array}$ \\
\hline 8 & 0.398557 & - \\
16 & 0.485816 & 21.89 \\
24 & 0.503804 & 3.70 \\
48 & 0.517342 & 4.67 \\
60 & 0.526165 & 1.17 \\
80 & 0.529840 & 0.70 \\
98 & 0.531862 & 0.38 \\
120 & 0.533538 & 0.31 \\
140 & 0.534578 & 0.16 \\
\hline
\end{tabular}

At this stage, a beam model, composed of 98 elements, could be considered as the near optimum, the top discretization beam model. For that discretization a numerical convergence is obtained with an error approach less than $0.5 \%$, accepted as 
top tolerance. In account to obtaining free vibration dynamic response curves of shear sensibility of beams, relations between the ratio of adimensional transversal displacement $\mathrm{w}(\mathrm{G} 0)$ to $\mathrm{w}(\mathrm{G} 4)$ versus aspect ratio, h/L, coefficient, were plotted in fig. 1. In that relation $\mathrm{w}(\mathrm{G} 0)$ is relative to the first mode maximum transversal displacement obtained from simulations with consideration of shear deformability, and $\mathrm{w}(\mathrm{G} 4)$ denotes those obtained without consideration of that effect, simulated with the product of the shear modulus, $\mathrm{G}$, by a power index of $10^{4}$, verified as an optimum, as done by Pietrobon [17] in 1998. This approach is the second analysis stage in which maximum transversal displacements, w, was computed for the discrete node of maximum dynamic deflection, relatively to each beam model. At this stage, (ss), (sc) and (cc), beams models were considered, for beams spans between $200 \mathrm{~nm}$ and $1500 \mathrm{~nm}$. Numerical solutions were plotted in fig. 1, a figure of curves pattern, as curves of sensibility coefficient, $\eta$, of Timoshenko beams models to shear

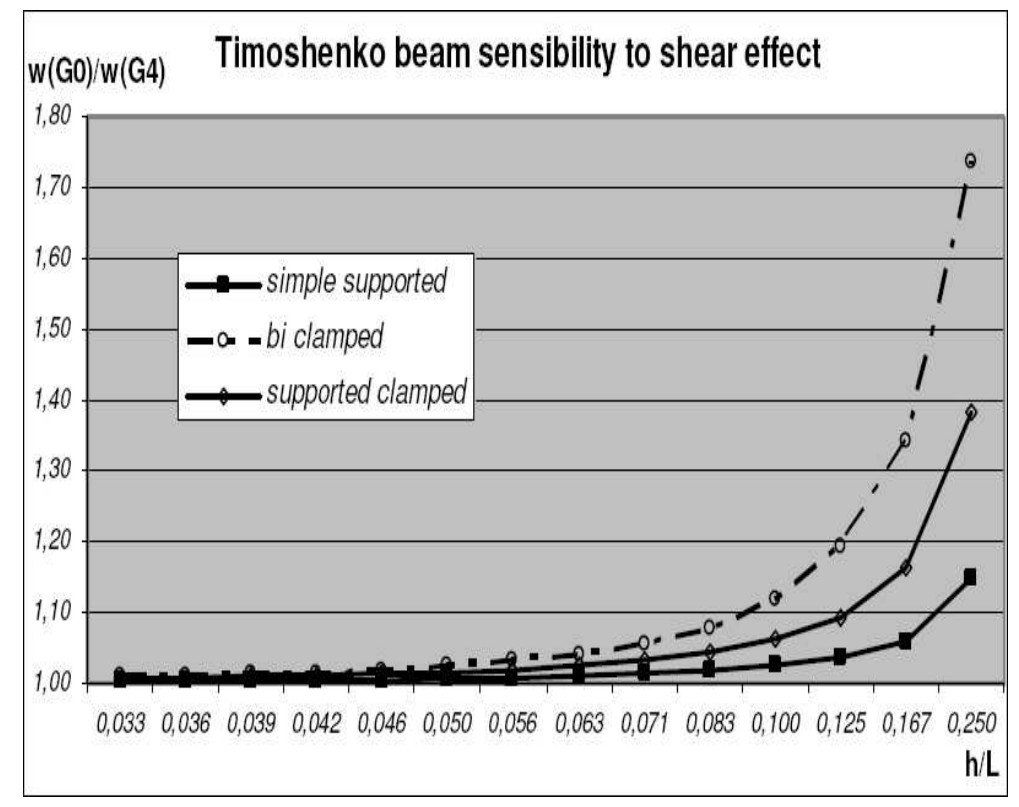

Figure 1: Sensibility coefficient - curves pattern

deformability effect. Results are curves of $\eta$ patterns, taking in account boundary conditions, for simple supported (ss), supported-clamped (sc) and bi-clamped (or bi-fixed), (cc) beams. Those curves show a increment in transversal displacement as aspect ratio increases. To specify the limit of validity to Timoshenko Beam Theory, numerical values of sensibility coefficient were processed, as postulated in this work. Values are presented in table 2, related to aspect ratio, as follows: the first column contains aspect ratio, in the the $2^{\text {nd }}$ to $4^{\text {th }}$ columns are numeric values of $\eta$ coefficient, and $5^{\text {th }}$ to $7^{\text {th }}$ columns lists values of adimensional percent $\left(\mathrm{x} 10^{-2}\right)$ transversal relative deflection of the beam. Numerical (computational) results have a significant variation from a beam model to another, as a function of the boundary 
Table 2 Computational results for (a) sensibility coefficient to shear effect, $2^{\text {nd }}$ to $4^{\text {th }}$ columns, and (d) adimensional growth in transversal displacement, $5^{\text {th }}$ to $7^{\text {th }}$ columns

\begin{tabular}{|c|c|c|c|c|c|c|}
\hline Aspect & \multicolumn{3}{|c|}{$\eta$ curves pattern } & \multicolumn{3}{|c|}{$\mathrm{w}(\mathrm{G} 0) / \mathrm{w}(\mathrm{G} 4)$} \\
\hline ratio & Model & Model & Model & Model & Model & Model \\
\hline$(h / L)$ & $s s$ & $s c$ & $c c$ & $s s$ & $s c$ & $c c$ \\
\hline 0.033 & 0.145081 & 0.353132 & 0.545837 & 1.002980 & 1.007262 & 1.010093 \\
\hline 0.036 & 0.180411 & 0.437255 & 0.649064 & 1.003415 & 1.008322 & 1.011730 \\
\hline 0.039 & 0.231243 & 0.552648 & 0.764001 & 1.003957 & 1.009633 & 1.013677 \\
\hline 0.042 & 0.214034 & 0.534128 & 0.790227 & 1.004650 & 1.011291 & 1.015969 \\
\hline 0.046 & 0.300111 & 0.701071 & 1.343190 & 1.005506 & 1.013428 & 1.019130 \\
\hline 0.050 & 0.293272 & 0.623192 & 1.194331 & 1.006707 & 1.016232 & 1.024503 \\
\hline 0.056 & 0.365224 & 0.720132 & 1.372464 & 1.008466 & 1.019971 & 1.031669 \\
\hline 0.063 & 0.414671 & 0.906250 & 1.674238 & 1.011023 & 1.025012 & 1.041276 \\
\hline 0.071 & 0.346905 & 0.990484 & 1.902693 & 1.014340 & 1.032262 & 1.054670 \\
\hline 0.083 & 0.419529 & 1.072729 & 2.547448 & 1.018503 & 1.044148 & 1.077503 \\
\hline 0.100 & 0.418573 & 1.225833 & 2.893239 & 1.025635 & 1.062384 & 1.120809 \\
\hline 0.125 & 0.534719 & 1.667606 & 3.585939 & 1.036100 & 1.093030 & 1.193140 \\
\hline 0.167 & 1.077178 & 2.633718 & 4.730563 & 1.058558 & 1.163070 & 1.343750 \\
\hline 0.250 & 1.683263 & 4.341721 & 8.324396 & 1.147964 & 1.381668 & 1.736386 \\
\hline
\end{tabular}

condition associated with support restrictions, as aspect ratio increases.

A crossing of data from this last table allows a verification of the growth of magnitudes in transversal displacement obtained from Timoshenko's Theory in relation to that obtained with employment of Euler-Bernoulli's one. The following percentages were obtained from the three last columns of table 2 with the transformation of those numerical results, $\varphi$ in general, for percent data: $(\varphi-1.0) \times 100 \%$. For a first view, applying Timoshenko's Beam Theory to CNT, in a way to determine a superior range of validation to TBT, using the aspect ratio less of 0.100 specified by Harik (2001) for take in account shear effects, is equivalent to accept a growth greater then $2.56 \%$, in displacement with consideration of shear effect, for simplesupported beams, relatively to that obtained without this approach, resulting for supported-clamped beams and bi-clamped, a growth around $6.23 \%$, and of 12.08 $\%$, respectively. In another view, admitting a top tolerance in displacement values growth less than $2.5 \%$ level, as a result of TBT employment, $\eta$, propose a frontier value between .419529 , for simple supported beams, 1.194331, for bi-clamped beams, and stay at 0.906250 , for supported-clamped beams. The values proposed by Harik correspond to growth in transversal displacement, w, between TBT and EBT from $5.85 \%$ to $34.37 \%$, high values for any analysis. This poses a strong recommendation: don't avoid shear effect. The use of $\eta$ coefficient as established in this work is a potential design support to predict lateral deflections of nano-beams. 


\section{Conclusions}

In numerical solutions, developed with computational simulations, it was verified exponential growing in transversal displacement as aspect ratio increases. That growth in dynamic deflection is associated to displacement verified for Timoshenko's beams models when compared to those obtained without consideration of shear correction, as Euler-Bernoulli Theory does. Curves of shear sensibility was developed as a ratio of adimensional deflection, as a relation of transversal displacements relating both those beams theories, to aspect ratio, a geometric index. Numerical results obtained with the concept of a shear sensibility coefficient supplies a proposition for the validity of Timoshenko's Beam Theory, and a design coefficient for employment in carbon nanotubes project. The aspect ratio around 0.100 proposed for Harik to CNT design corresponds to an increase in transversal displacement of $2.56 \%$, for simple supported beams. To acquire an analogous increase in transversal displacement of the beam model, it is needed to project an aspect ratio of 0.063 , for supported-clamped boundary restriction, and between 0.05, for bi-clamped, models. The sensibility coefficient to shear effect may support the design of deflections of nano-beams.

\section{References}

[1] H. Antes, M. Schanz, S. Alvermann, Dynamic analyses of plane frames by integral equations for bars and Timoshenko beams, Journal of Sound and Vibrations, 276, No. 3-5 (2004), 807 - 836.

[2] D.N. Arnold, A.L. Madureira, S. Zhang, On the range of applicability of the Reissner-Mindlin and Kirchhoff-Love plate bending models, Journal of Elasticity, 67, No. 3 (2002), 171 - 185.

[3] M.J. Beran, The use of classical beams theory for micro-beams composed of polycristals, International Journal of Solids and Structures, 35, No. 19 (1998), 2407-2412.

[4] G.R. Cowper, The shear coefficient in Timoshenko's beam theory, Journal of Applied Mechanics 33, No. 2 (1966) (Trans. ASME 88 E.)

[5] J. Donea, A. Huerta, "Finite Element Methods for Flow Problems", John Wiley and Sons, 2003.

[6] L. Formmagia, J.F. Gerbeau, F. Nobili, A. Quarteroni, On the coupling of 3D and 1D Navier-Stokes equations for flow problems in compliant vessels, Compute Methods in Applies Mech. Engineering, 191 (2001), 561-582.

[7] Frankland et al., "The Stress-strain Behavior of Polymer-nanotube Composites from Molecular Dynamics Simulations", Langley Research Center, ICASE Report No. 2002-41, 2002.

[8] L. Garcia, F. Taborda, S.F. Villaça, "Teoria da Elasticidade", COPPE-UFRJ, 2000 . 
[9] V.M. Harik, Ranges of applicability for the continuum beam model in the constitutive analysis of carbon nanotubes: nanotubes or nano-bems?, NASA ICASE Thecnical Report, No. 2001-16, 2001.

[10] J.T.R. Hughes, "The Finite Element Method", Prentice-Hall Inc., 1995.

[11] H. Katori, Considerations of the problem of shearing and torsion of thin-walled beams with arbitrary cross section, Thin-Walled Structures, 39 (2001), 671684 .

[12] M. Krommer, H. Irschik, On the influence of coupling between electrical and mechanical fields upon the flexural behavior of smart composite beams, in "Annals of ECCOMAS", 2000.

[13] M. Levinson, D.W. Cook, Thick rectangular plates - I: The generalized Navier solution, International Journal of Mechanics Science, 25, No. 3 (1983).

[14] M. Levinson, D.W. Cook, Thick rectangular plates - II: The generalized Lévy solution, International Journal of Mechanics Science, 25, No. 3 (1983).

[15] R.D. Mindlin, Influence of rotatory inertia and shear on flexural motions of isotropic elastic plates, Journal of Applied Mechanics, 3 (1951).

[16] F.C. Pietrobon, Impacto do coeficiente de cisalhamento na flexão dinâmica de vigas, Revista Engenharia Ciência Tecnologia, UFES, 6, No. 1 (2003), 21-30.

[17] F.C. Pietrobon, "Análise Numérica da Flexão Dinâmica de Vigas com a Consideração da Deformabilidade por Cortante e da Inércia de Rotação", Tese de Mestrado, PEC/COPPE/UFRJ, Rio de Janeiro, RJ, 1998.

[18] S.S. Rao, "Mechanical Vibrations", Addison-Wesley Publishing Co. 3rd ed., 1995.

[19] E. Reissner, The effect of transverse shear deformation on the bending of elastic plates, Journal of Applied Mechanics, 6 (1945).

[20] I.H. Shames, "Mechanics of Deformable Solids", Prentice Hall Inc., 1964.

[21] C.W. Silva, Dynamic beam model with internal damping rotatory inertia and shear deformation, AIAA Journal, 12, No. 5 (1976).

[22] D. Sun, L. Tong, S.N. Atturi, Effects of piezoletectric sensors/actuators debonging on vibration control of smart beams, International Journal of Solids and Structures, 38, No. 50-51 (2001), 9033-9051.

[23] S. Timoshenko, On the vibration of bars of uniform cross section, Philosophycal Magazine, 43, No. 6 (1922), 125-131.

[24] C.M. Wang, V.B.C. Tan, Y.Y. Zhang, Timoshenko beam model for vibration analysis of multi-walled carbon nanotubes, Journal of Sound and Vibration, 294 (2006), 1060-1072. 